\title{
Liposomes as nanoreactors for the photochemical synthesis of gold nanoparticles
}

\author{
Sushanth Gudlur, Camilla Sandén, Petra Matouskova, Chiara Fasciani and Daniel Aili
}

\section{Linköping University Post Print}

\section{Tweet}

N.B.: When citing this work, cite the original article.

Original Publication:

Sushanth Gudlur, Camilla Sandén, Petra Matouskova, Chiara Fasciani and Daniel Aili, Liposomes as nanoreactors for the photochemical synthesis of gold nanoparticles, 2015, Journal of Colloid and Interface Science, (456), 206-209.

http://dx.doi.org/10.1016/j.jcis.2015.06.033

Copyright: Elsevier

http://www.elsevier.com/

Postprint available at: Linköping University Electronic Press

http://urn.kb.se/resolve?urn=urn:nbn:se:liu:diva-120718 


\title{
Liposomes as nanoreactors for the photochemical synthesis of gold
}

\section{nanoparticles}

\author{
Sushanth Gudlur ${ }^{a, b}$, Camilla Sandén $^{a}$, Petra Matoušková ${ }^{a, c}$, Chiara Fasciani ${ }^{a, d}$ and \\ Daniel Aili ${ }^{a^{*}}$
}

A simple and novel method for the photochemical synthesis of AuNPs in liposomes is described. Gold salt is coencanpsulated with the photoinitiator Irgacure-2959 in POPC liposomes prepared via traditional thin-film hydration technique. UVA irradiation for 15 minutes results in encapsulated AuNPs of $2.8 \pm 1.6 \mathrm{~nm}$ in diameter that are primarily dispersed in the aqueous interior of the liposomes.

Gold nanoparticles (AuNPs) have widespread applications in plasmonics, ${ }^{1}$ biosensors, ${ }^{2}$ electronics, ${ }^{3}$ catalysis, ${ }^{4}$ imaging and drug delivery. ${ }^{5,6}$ A large number of methods have been developed and optimized for synthesizing AuNPs with defined sizes and shapes, ${ }^{7,8}$ and typically involves the chemical reduction of $\mathrm{Au}$ (III) to $\mathrm{Au}(0)$. The initial small clusters of reduced gold thus obtained serve as nucleation sites for the subsequent growth of nanoparticles. For synthesis of spherical AuNPs, besides a reducing agent, an additional stabilizing or capping agent is sometimes added to the reaction mixture to prevent the AuNPs from aggregating. McGilvray et al. (2006), ${ }^{9}$ reported a simple and rapid photochemical synthesis of AuNPs to obtain monodisperse spherical nanoparticles that are stable in suspension. In this synthesis, the reduction of $\mathrm{Au}$ (III) was achieved by ketyl radicals generated due to the UVA-irradiation of Irgacure-2959 (Irg), a photoinitiator. Additionally, the 4hydroxyethoxy benzoic acid generated from irradiated Irg eventually acts to stabilize the AuNPs and thus prevents them from aggregating.

Liposomes are excellent candidates for the compartmentalized synthesis of AuNPs, ${ }^{10}$ especially if the end-applications are in the field of biological probes and drug delivery. Such compartmentalized synthesis offers the possibility to: control particle size, control the environment of synthesis to include catalytically active components, and carry out synthesis in a highly organized solvent structure. ${ }^{11}$ Yet, despite such advantages, previous attempts at synthesizing AuNPs within liposomes have resulted in high size polydispersity, inhomogeneity in shape, or poor yield. ${ }^{12-14}$ Recently, the synthesis of AuNPs within liposomes was achieved by the controlled diffusion of stabilizing agents into encapsulated gold salt solutions, ${ }^{15,16}$ or by doping the lipids that constitute a liposome with glycerol that acts as a reducing agent. ${ }^{17}$ However, these approaches have had the need to either modify the method of liposome preparation or use special lipids to achieve controlled synthesis of AuNPs.

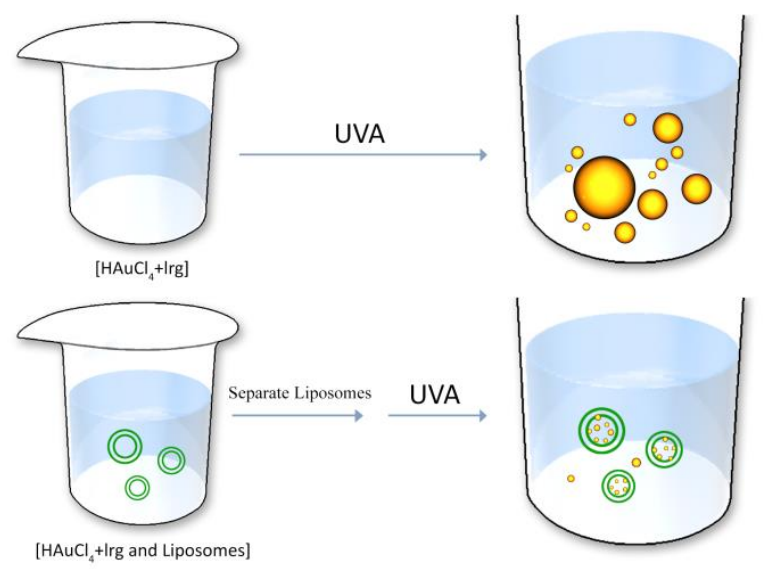

Fig. 1 Schematic illustration of the photochemical synthesis of AuNPs in bulk solvent (top panel) and within liposomes (bottom panel). Green circles represent liposomes and golden spheres represent AuNPs.

In this communication, we describe a simple method (Fig.1) for the controlled synthesis of spherical AuNPs with an average size of 2.8 $\pm 1.6 \mathrm{~nm}$, inside palmitoyl oleoyl phosphocholine (POPC) liposomes. The resulting AuNPs are homogeneous in size and shape. Moreover, this simple method circumvents the need for separating unencapsulated AuNPs after their synthesis, which can be challenging when employing the more traditional method of encapsulating preformed AuNPs within liposomes. All these, without having to (1) modify existing liposome preparation techniques, (2) introduce additional stabilizing agents in solution, or (3) dope/modify lipids that constitute a liposome, with reducing or stabilizing agents. 
A freshly prepared solution of $\mathrm{HAuCl}_{4}(0.1 \mathrm{mg} / \mathrm{mL})$ and $\operatorname{Irg}(1 \mathrm{mM})$ in deionized water was encapsulated into POPC liposomes (2 $\mathrm{mg} / \mathrm{mL}$ ) obtained via the thin-film hydration technique ${ }^{18}$ followed by extrusion using a polycarbonate filter of $100 \mathrm{~nm}$ pore size. After removal of unencapsulated $\mathrm{HAuCl}_{4}$ and $\mathrm{Irg}$ by separating them over a Sephadex G-50 spin column, the samples were irradiated in a photoreactor with $108 \mu \mathrm{W} / \mathrm{m} 2$ UVA light $(365 \mathrm{~nm})$ for $15 \mathrm{mins}$, to generate stable AuNPs within the confines of the liposome.

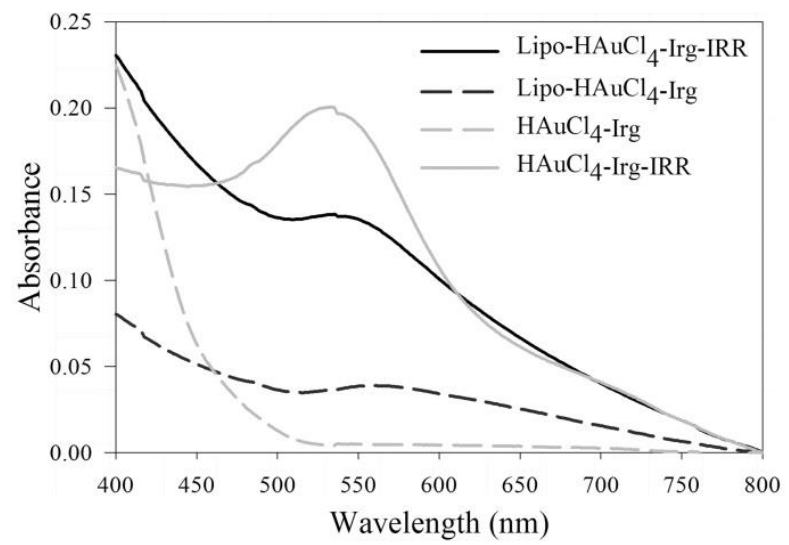

Fig. 2 UV-Vis spectra of $0.1 \mathrm{mg} / \mathrm{mL} \mathrm{HAuCl}_{4}+1 \mathrm{mM} \mathrm{Irg} \mathrm{samples}$ unencapsulated (gray lines) or encapsulated within POPC liposomes (black lines). Samples were prepared in the dark (dashed lines) followed by UVAirradiation (IRR) for 15 mins (solid lines) to initiate AuNP synthesis.

Photochemical synthesis of AuNPs within liposomes was confirmed by the presence of the localized surface plasmon resonance (LSPR) band centered around $530 \mathrm{~nm}$ (Fig. 2). While this LSPR band is typically centered around $520 \mathrm{~nm}$ for small $(\sim 10 \mathrm{~nm})$ and dispersed AuNPs, its position is affected by factors such as particle size, shape, refractive index of the medium, temperature and by species adsorbed on the nanoparticle surface ${ }^{1}$. The position of the LSPR band for AuNPs within liposomes appears red shifted due to the presence of liposomes that scatter significantly at lower wavelengths. The effect of liposome scattering on the LSPR peak position was confirmed using liposome encapsulation of commercial AuNPs. In the absence of liposomes they show $\lambda$ max at $\sim 520 \mathrm{~nm}$, and when encapuslated, the peak shifted to $\lambda$ max $\sim 550 \mathrm{~nm}$ (Fig. S1). On the other hand, very small AuNPs $(<2 \mathrm{~nm})$ lose their plasmonic properties and hence will not show an LSPR band. ${ }^{19-21}$ This loss of plasmonic properties may explain to some extent, the relatively weaker LSPR band from samples where AuNPs were synthesized within liposomes.

Interestingly, transmission electron microscopy (TEM) revealed significant differences in nanoparticle size and the overall size distribution of the AuNPs synthesized within liposomes and in bulk solvent (Fig. 3) using otherwise identical conditions. The AuNPs synthesized within liposomes are very homogeneous in shape and size (Fig. 3A). Based on the TEMs and size distribution analysis performed on these images, the AuNPs are spherical and have an average size of $2.8 \pm 1.6 \mathrm{~nm}$ (Fig. $3 \mathrm{~A}$ and $4 \mathrm{~A}$ ). In comparison, there is a significant difference in the size range of AuNPs synthesized in bulk solvent when using the same conditions but excluding liposomes (Fig. 3B and 4A). These nanoparticles are significantly larger in size and more polydispersed.

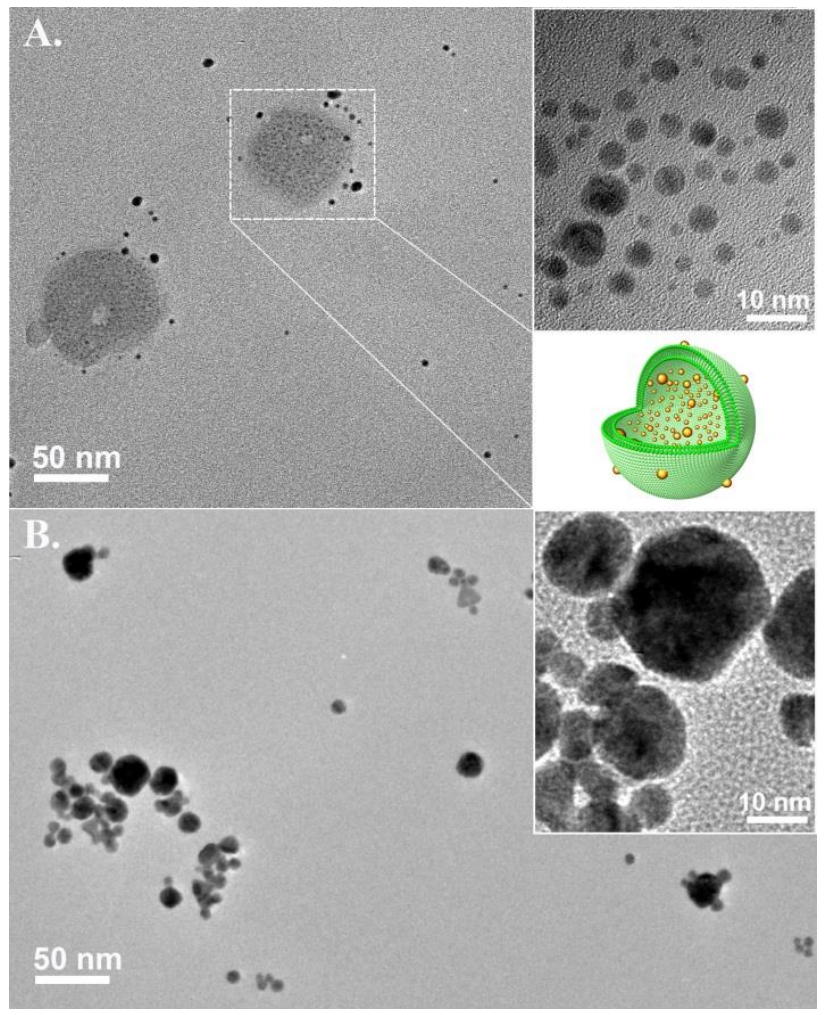

Fig. 3 TEM Characterization of AuNPs synthesized within POPC liposomes (A) or in bulk solvent (B). Insets are magnified images from the same samples. Magnification in Inset A is a portion of the liposome. Colored inset in (A) is a representation of AuNPs within liposomes.

The large difference in the AuNP sizes between those synthesized within liposomes and those in the bulk solvent is presumably due to an effect of confinement as well as the presence of a lipid bilayer. The inner membrane of the liposome offers a convenient surface for adsorption, formation and growth of nuclei but affects the diffusion coefficient of the nuclei. ${ }^{22,23}$ Hence, when compared to the bulk solvent, the nuclei formation within the liposomes is fast but the smaller diffusion coefficient of the nuclei along with the physical barrier which inhibits NP diffusion, encounter and agglomeration, slows down the rate of the particle growth. ${ }^{24}$ Such reduced growth rates allow for other processes such as the capping/stabilization of AuNPs to occur and thus limits and controls the size of the particles formed.

We investigated the effect of $\mathrm{HAuCl}_{4}+$ Irg concentrations and time of irradiation on AuNP synthesis in suspension (Fig. S2). We chose the minimum concentration and time of irradiation required to synthesize stable AuNPs, for synthesis within POPC liposomes. This was done to avoid any effects of AuNP aggregation due to high concentration of reactants and lipid peroxidation due to excessive UVA irradiation. 
A.

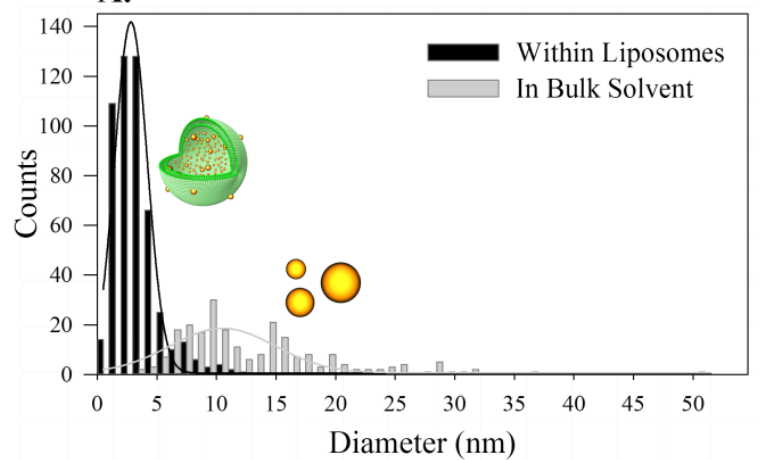

B.

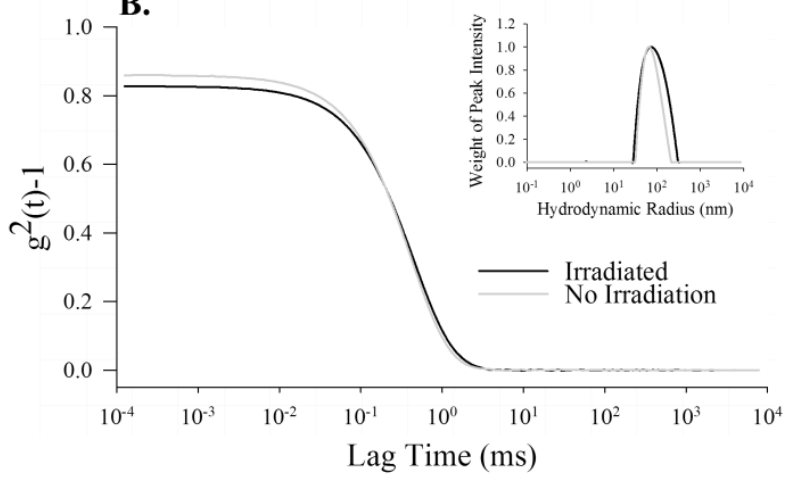

Fig. 4 (A) Size distribution analysis of particles synthesized with $0.1 \mathrm{mg} / \mathrm{mL}$ $\mathrm{HAuCl}_{4}+1 \mathrm{mM}$ Irg in bulk solvent (gray) where $\mathrm{n}=231$, and encapsulated in POPC liposomes (black) where $\mathrm{n}=509$. (B) DLS correlation curve for encapsulated samples prepared with $0.1 \mathrm{mg} / \mathrm{mL} \mathrm{HAuCl} 4+1 \mathrm{mM} \mathrm{Irg}$ before (gray) and after (black) UVA-irradiation. The inset shows size distribution obtained using the CONTIN algorithm. POPC Liposomes retain their size to a large extent after irradiation.

In the case of AuNPs synthesized within liposomes, from the TEMs alone, it is difficult to ascertain the exact location of the AuNPs. Besides being localized within the aqueous region of the liposome, the AuNPs, to a lesser extent, are expected to be trapped both on the periphery (via electrostatic interaction) as well as within the bilayer. Lipid peroxidation is expected to contribute to some reduction within the bilayer or in its vicinity. ${ }^{13,14}$ It is known that certain lipids are able to spontaneously reduce $\mathrm{Au}$ (III) to $\mathrm{Au}(0)$ through the oxidation of the $-\mathrm{OH}$ groups to $-\mathrm{COOH}$ groups. ${ }^{25}$ This type of reduction may explain the presence of a weak LSPR band in our liposome samples when the samples were prepared in the dark and in the absence of UVA-irradiation (Fig. 2, black-dashed line). The LSPR band is absent when control $\mathrm{HAuCl}_{4}$ solution containing Irg was prepared in the dark and not irradiated (Fig. 2, gray-dashed line). Additionally, the presence of Irg and the subsequent UVAirradiation of the liposomes can induce an oxidative stress leading to some lipid peroxidation, which cannot be avoided given the photochemical method of this AuNP synthesis. From the TEMs (Fig. $3 \mathrm{~A}$ ), it can be seen that some AuNPs are present outside the liposomes. This is most likely due to a combination of (1) the diffusion of trace amounts of the gold from within the liposomes during the separation/purification stage (2) due to normal liposomal leakage and/ or (3) disruption of liposomes during TEM sample preparation.
To investigate the effect of UV irradiation on the liposomes, samples were analyzed with dynamic light scattering (DLS). The DLS instrument consisted of a ALV-CGS-5022F Goniometer system, a $\mathrm{HeNe}$ laser and a dual multiple-tau digital correlator (ALV-6010160). For each sample, the count rate was approximately $350 \mathrm{kHz}$ with a measuring time of $10 \times 30$ s. Correlation curves and calculated size distributions were compared and showed little change for all liposome containing samples (Fig. 4B). The liposomes retain their size which is a good indication that they also retain their integrity.

\section{Conclusion}

Taken together, these results indicate that it is possible to synthesize AuNPs of homogeneous size distribution within liposomes without the need to modify existing methods of liposome preparation. We envision that this method of AuNP synthesis can be extended to other methods of liposome preparation as well. While we have demonstrated the controlled synthesis of AuNPs in liposomes prepared from POPC, which are already in the liquid-crystalline state at room temperature, this can be potentially expanded to liposomes prepared with other phospholipids having different gel-to-crystalline phase transition temperatures. The choice of lipids will therefore dictate the stability and release pattern-which is an important trigger control when it comes to designing suitable drug delivery systems. AuNPs within liposomes have applications as probes in biological processes wherein, the course and fate of the liposomes within the cell or body can be followed over time. In the case of drug delivery, the release of the contents of such liposomes can be triggered by thermal ablation via plasmon excitation of AuNPs. While this method may not be suitable for all drugs or excipients being encapsulated in liposomes, because of the drug's interaction with AuNPs or the drug's stability towards UVA-irradiation, it can prove useful in many other instances.

An alternative strategy to obtain liposome encapsulated AuNPs is by encapsulating pre-formed AuNPs into liposomes. Here, a thin film of dried lipids is hydrated with pre-formed AuNPs in suspension. After extruding the liposomes, separation of unencapsulated AuNPs is achieved via size exclusion chromatography or centrifugation. Due to the small difference in their sizes (AuNPs and liposomes), size exclusion chromatography is more challenging while centrifugation is time consuming. Using the method described in this communication, this step can be circumvented since the separation step is performed before the AuNPs are synthesized. Removing unencapsulated gold salt solution using Sephadex G-50 spin columns is both easier and faster than removing unencapsulated AuNPs.

Acknowledgements: This work was supported by the Swedish Foundation for Strategic Research (SSF) and the Center for Integrative Regenerative Medicine (IGEN) at Linköping University. During this study C.S. was enrolled in the graduate school Forum Scientium. The authors thank Batika Saxena for creating the artwork.

\section{Notes and references}

${ }^{a}$ Division of Molecular Physics, Department of Physics, Chemistry and Biology (IFM), Linköping University, SE-583 36 Linköping, Sweden.

* Corresponding Author: daniel.aili@liu.se

b Current Address: Centre for Biomimetic Sensor Science, School of Materials Science and Engineering, Nanyang Technological University, Singapore 637553 
${ }^{\mathrm{c}}$ Current Address: Centre for Materials Research, Faculty of Chemistry, Brno University of Technology, Purkynova 118, 61200 Brno, Czech Republic.

d Current Address: Istituto Italiano di Tecnologia, Viale Regina Elena 291, 00161 Rome, Italy.

${ }^{\dagger}$ Electronic Supplementary Information (ESI) available

1. C. J. Orendorff, T. K. Sau and C. J. Murphy, Small, 2006, 2, 636.

2. S. Szunerits and R. Boukherroub, Chem. Commun., 2012, 48, 8999.

3. D. Huang, F. Liao, S. Molesa, D. Redinger and V. Subramanian, J. Electrochem. Soc., 2003, 150, G412.

4. B. Hvolbæk, T. V. W. Janssens, B. S. Clausen, H. Falsig, C. H. Christensen and J. K. Nørskov, Nano Today, 2007, 2, 14.

5. T. Curry, R. Kopelman, M. Shilo and R. Popovtzer, Contrast Media Mol. Imaging, 2014, 9, 53.

6. R. A. Sperling, P. Rivera Gil, F. Zhang, M. Zanella and W. J. Parak, Chem. Soc. Rev., 2008, 37, 1896.

7. P. Zhao, N. Li and D. Astruc, Coord. Chem. Rev., 2013, 257, 638.

8. M. C. Daniel and D. Astruc, Chem. Rev., 2004, 104, 293.

9. K. L. McGilvray, M. R. Decan, D. Wang and J. C. Scaiano, J. Am. Chem. Soc., 2006, 128, 15980.

10. P. He and M. W. Urban, Biomacromolecules, 2005, 6, 1224.

11. D. G. Shchukin and G. B. Sukhorukov, Adv. Mater., 2004, 16, 671.

12. G. M. Chow, M. A. Markowitz, R. Rayne, D. N. Dunn and A. Singh, J. Colloid Interface Sci., 1996, 183, 135.

13. F. C. Meldrum, B. R. Heywood and S. Mann, J. Colloid Interface Sci., 1993, 161, 66.

14. O. Regev, R. Backov and C. Faure, Chem. Mater., 2004, 16, 5280.

15. T. K. Sau, A. S. Urban, S. K. Dondapati, M. Fedoruk, M. R. Horton, A. L. Rogach, F. D. Stefani, J. O. Rädler and J. Feldmann, Colloids Surf. A: Physicochem. Eng. Asp., 2009, 342, 92.

16. R. Genc, M. Ortiz and C. Sullivan, J. Nanopart. Res., 2014, 16, 1.

17. R. Genc, G. Clergeaud, M. Ortiz and C. K. O'Sullivan, Langmuir, 2011, 27, 10894.

18. A. D. Bangham and R. W. Horne, J. Mol. Biol., 1964, 8, 660.

19. Z. Y. Li, J. P. Wilcoxon, F. Yin, Y. Chen, R. E. Palmer, R. L. Johnston, Faraday Discuss., 2008, 138, 363.

20. S. Gilb, K. Hartl, A. Kartouzian, J. Peter, U. Heiz, H. -. Boyen and P. Ziemann, Eur. Phys. J. D, 2007, 45, 501.

21. H. -. Weissker and C. Mottet, Phys. Rev. B, 2011, 84, 165443.

22. T. V. Liveri, ed. J. David Lockwood. 2006. ch. 2, pp. 75.

23. M. T. Kennedy, B. A. Korgel, H. G. Monbouquette and J. A. Zasadzinski, Chem. Mater., 1998, 10, 2116.

24. C. Tojo, M. C. Blanco, F. Rivadulla and M. A. LÃ ${ }^{3}$ pez-Quintela, Langmuir, 1997, 13, 1970.

25. K. Esumi, T. Hosoya, A. Suzuki and K. Torigoe, Langmuir, 2000, 16, 2978. 
Electronic Supplementary Information

\title{
Liposomes as Nanoreactors for the Photochemical Synthesis of Gold Nanoparticles
}

\author{
Sushanth Gudlur ${ }^{a, b}$, Camilla Sandén $^{a}$, Petra Matouškováa ${ }^{a, c}$, Chiara Fasciani $^{a, d}$ and Daniel Aili ${ }^{a^{*}}$
}

\begin{abstract}
Materials
1-palmitoyl-2-oleoyl-sn-glycero-3-phosphocholine (16:0-18:1) was purchased from Avanti Polar Lipids, Inc (AL, USA). Gold (III) chloride hydrate and 2-Hydroxy-4'-(2-hydroxyethoxy)-2-methylpropiophenone (Irgacure-2959) was purchased from Sigma Aldrich (Sweden). All UV-vis absorbance scans were obtained on a Shimadzu UV-2450, UV-Vis Spectrophotometer. TEM images were acquired using a Tecnai G2 F20U-Twin microscope operating at $200 \mathrm{kV}$. $15 \mathrm{~nm}$ AuNPs were purchased from BBI Solutions (Cardiff, UK).
\end{abstract}

\section{AuNP synthesis in POPC liposomes}

$80 \mu \mathrm{L}$ of POPC $(25 \mathrm{mg} / \mathrm{mL})$ in chloroform was dried to a thin film in a $10 \mathrm{~mL}$ conical flask under a constant stream of nitrogen. The dried lipids were placed under vacuum for $2 \mathrm{hrs}$ to remove residual chloroform. Meanwhile, a $1 \mathrm{mg} / \mathrm{mL}$ solution of $\mathrm{HAuCl}_{4}$ was freshly prepared in DI water and diluted into a freshly prepared solution of Irgacure - 2959 (Irg) such that, the final concentration of HAuCl4 and Irg was $0.1 \mathrm{mg} / \mathrm{mL}$ and $10 \mathrm{mM}$ respectively. Preparation and mixing of the various solutions were carried out in the dark (or under minimal light) and covered with aluminum foil to protect from stray light. This $\mathrm{HAuCl}_{4}+\mathrm{Irg}$ was added to the thin film of dried lipids to a final lipid concentration of $2 \mathrm{mg} / \mathrm{mL}$ and vortexed vigorously for $1 \mathrm{hr}$ on a shaker. The resulting samples were then extruded 21 times through a polycarbonate filter with $100 \mathrm{~nm}$ pore size. After extrusion, the samples were passed through a Sephadex G-50 spin column by centrifuging them once at $1500 \mathrm{rpm}$ for 3 mins. After removal of unencapsulated $\mathrm{HAuCl}_{4}$ and Irg, the samples were irradiated in a photoreactor with $108 \mu \mathrm{W} / \mathrm{m} 2$ UVA light (365 nm) for 15 mins, to generate stable AuNPs. These samples were used to collect absorbance, DLS and TEM images.

\section{Effect of Light Scattering on LSPR Peak Position}

The LSPR peak position of commercial AuNPs $(15 \mathrm{~nm})$ in POPC liposomes is slightly red shifted when compared to commercial AuNPs dispersed in buffer (Fig S1, dashed lines). A similar red shift is also observed when comparing LSPR peaks of AuNPs synthesized in suspension and within POPC liposomes (Fig. S1, solid lines). Considering that the latter are significantly smaller than the commercial nanoparticles, and hence should be blue shifted, the observed red shift is most likely an effect of light scattering by POPC liposomes. 


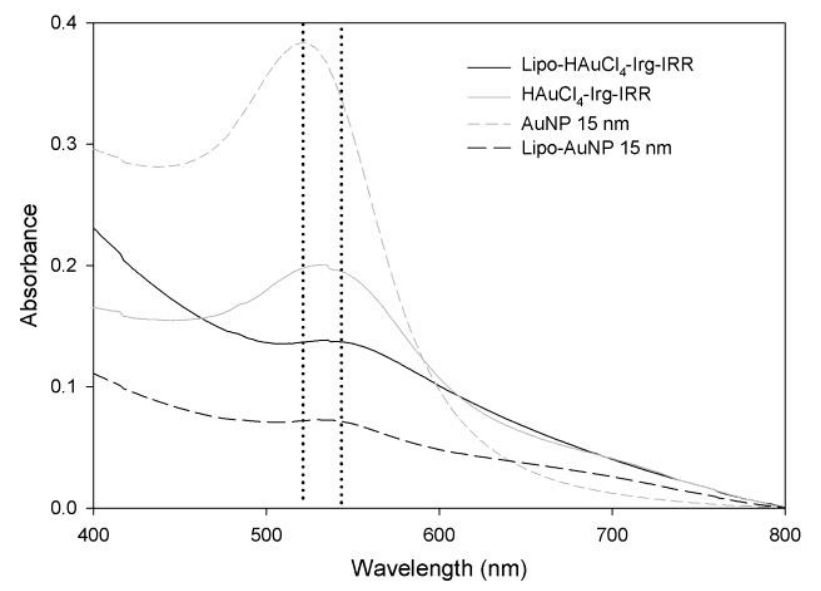

Fig. S1 Effect of light scattering on LSPR peak position. A comparison of the UV-Vis spectra of commercial AuNPs unencapsulated (grey dashed line) and encapsulated in POPC liposomes (black dashed line) shows a slight red shift in the LSPR peak position. A similar red shift can been seen for unencapsulated AuNPs prepared using 0.1 $\mathrm{mg} / \mathrm{mL} \mathrm{HAuCl}_{4}+1 \mathrm{mM}$ Irg (grey line) and when prepared within POPC liposomes (black line). Dotted vertical lines superimposed on the LSPR peaks are meant for ease of comparison of peak positions.

\section{Effect of concentration of $\mathrm{HAuCl}_{4}$ and time of irradiation on AuNP formation}

The synthesis of AuNPs in bulk solvent using the McGilvray method was monitoring using the intensity at $\lambda_{\max }$ of the LSPR band. Different concentrations of $\mathrm{HAuCl}_{4}$ (while maintaining a constant ratio of $\mathrm{HAuCl}_{4}$ : Irg) and time of irradiation were investigated. Successful synthesis was achieved at all concentrations used $(0.1 \mathrm{mg} / \mathrm{mL}-1 \mathrm{mg} / \mathrm{mL})$ and up to $60 \mathrm{mins}$ of irradiation.

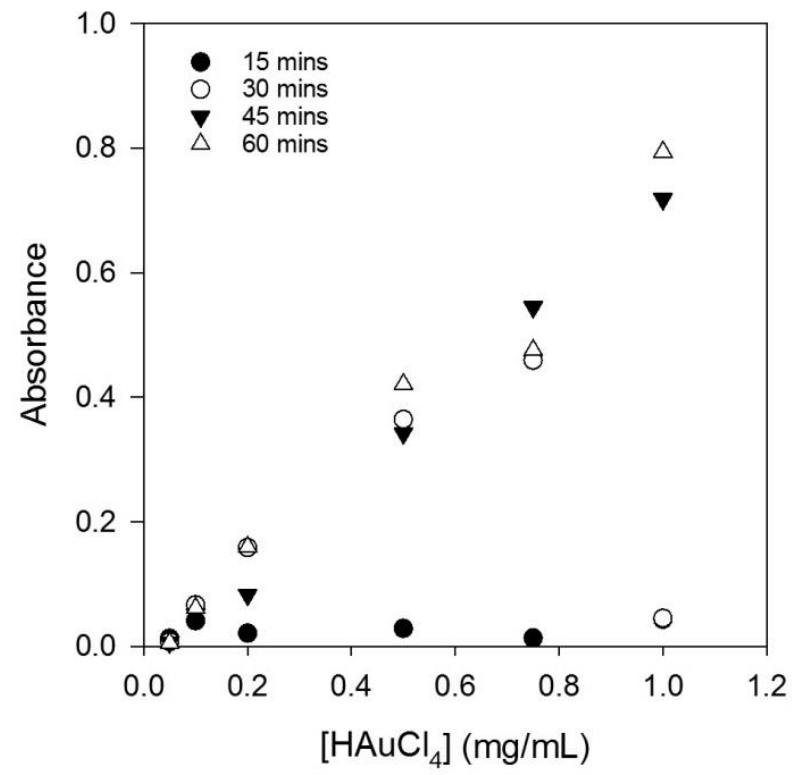

Fig. S2 Effect of $\mathrm{HAuCl}_{4}$ concentration and time of irradiation on AuNP synthesis as monitored by the intensity of the LSPR peak $\left(\lambda_{\max }\right)$. Synthesis was achieved at all concentrations used $(0.1 \mathrm{mg} / \mathrm{mL}-1 \mathrm{mg} / \mathrm{mL})$ and up to 60 mins of irradiation. 
${ }^{a}$ Division of Molecular Physics, Department of Physics, Chemistry and Biology (IFM), Linköping University, SE-583 36 Linköping, Sweden.

* Corresponding Author: daniel.aili@liu.se

b Current Address: Centre for Biomimetic Sensor Science, School of Materials Science and Engineering, Nanyang Technological University, Singapore 637553

${ }^{\mathrm{c}}$ Current Address: Centre for Materials Research, Faculty of Chemistry, Brno University of Technology, Purkynova 118,61200 Brno, Czech Republic.

${ }^{d}$ Current Address: Istituto Italiano di Tecnologia, Viale Regina Elena 291, 00161 Rome, Italy. 